\title{
Exploring the Activation of Semantic and Phonological Codes during Speech Planning with Event-Related Brain Potentials
}

\author{
Jörg D. Jescheniak ${ }^{1,2}$, Herbert Schriefers ${ }^{3}$, Merrill F. Garrett ${ }^{4}$, \\ and Angela D. Friederici ${ }^{1}$
}

\begin{abstract}
We present a new technique for studying the activation of semantic and phonological codes in speech planning using event-related brain potentials (ERPs) that extend a well-established behavioral procedure from speech production research. It combines a delayed picture-naming task with a priming procedure. While participants prepared the production of a depicted object's name, they heard an auditory target word. If the prepared picture name and the target word were semantically or phonologically related, the ERP waveform to the target word tended less towards
\end{abstract}

\section{INTRODUCTION}

When speaking, we bridge the cleft between preverbal thinking and articulatory motor programming, most of the time without much effort, at extremely high speed, and with surprisingly few errors (see Levelt, 1989, for a detailed treatment). A central component of this process is the selection of words appropriate for the expression of our communicative intention. As an example, if a speaker wants to talk about the head of the chicken yard, the word "rooster," rather than, say, the word "duck" needs to be retrieved from the speaker's mental lexicon. To date, detailed psycholinguistic models have been proposed describing the subprocesses of lexical access and their temporal dynamics, based on evidence from lexical retrieval failures and chronometric studies. Despite many yet unresolved issues in detail, there is a wide agreement on the basic distinction among access to a prelexical conceptual representation, access to a lexical lemma representation, and access to a lexical word-form representation (e.g., Levelt, Roelofs, \& Meyer, 1999; Dell \& O'Seaghdha, 1992; Garrett, 1988). The lemma is conceived as a nonphonological representation specifying either the word's syntactic (Levelt et al., 1999) or semantic and syntactic properties (Vigliocco, Vinson, Martin, \& Garrett, 1999).

\footnotetext{
${ }^{1}$ Max Planck Institute of Cognitive Neuroscience, ${ }^{2}$ University of Leipzig, ${ }^{3}$ Nijmegen University, ${ }^{4}$ University of Arizona
}

the negative when compared to an unrelated control. These effects were widely distributed. By contrast, if participants performed a nonlinguistic task on the depicted object (natural size judgment), the semantic effect was still obtained while the phonological effect disappeared. This suggests that the former effect indexes semantic activation involved in object processing while the latter effect indexes word-form activation specific to lexical processing. The data are discussed in the context of models of lexical access in speech production.

Only recently has the recording of event-related brain potentials (ERPs) been applied in the study of conceptual and lexical activation processes during speech production (Schmitt, Münte, \& Kutas, 2000; Abdel Rahman \& Sommer, 2000; van Turennout, Hagoort, \& Brown, 1997, 1998). Certainly, the major obstacle with respect to ERP research on speech production is that speaking is intrinsically linked to motor behavior whose reflections in the brain may easily outweigh and mask the reflections of the cognitive components one wants to study (e.g., Wohlert, 1993; Brooker \& Donald, 1980). Hence, experimental techniques need to be delineated which allow to dispense motor components while keeping the remaining process as similar as possible to normal speech production. In chronometric studies, picturenaming is a prominent and widely used experimental tool for studying lexical access. The present article presents a new ERP technique that is also based on picture naming. Before turning to a description of the experimental paradigm and the experimental data, we will briefly review ERP studies on lexical access available thus far.

\section{Lateralized Readiness Potential Studies in Language Production}

Most ERP studies on language production thus far used lateralized readiness potentials (LRPs) derived from the 
participant's electroencephalogram to explore the temporal sequence in which different types of lexical information can be retrieved during speech planning. The LRP is a specific index for response preparation and its onset provides an estimate for the moment in time at which a certain type of information affects the preparation of a voluntary movement response (e.g., Coles, Gratton, \& Donchin, 1988; de Jong, Wierda, Mulder, \& Mulder, 1988; Gratton, Coles, Sirevaag, Eriksen, \& Donchin, 1988).

In the study by van Turennout et al. (1997), participants' primary task was to name pictures of simple objects. On the critical trials, however, they were cued to postpone the naming response and to carry out a binary go/no-go decision first. They were asked to classify the depicted object along a semantic-conceptual dimension (animate vs. inanimate) and a phonological dimension (e.g., object name ending on the phoneme " $n$ " vs. object name ending on the phoneme "s"). The push-button response required was contingent on the outcome of the two classification tasks. In one version of the task, the semantic classification determined the response hand (e.g., right-hand response in the case of an animate object), while the phonological classification determined whether the response should be given or not (e.g., response only in the case of a word-final "n"). In the other version of the task, the phonological classification determined the response hand, while the semantic-conceptual classification determined whether a response had to be given or not.

If, in speech planning, semantic information is available prior to phonological information, preparation of the correct response in the first version of the task can be initiated as soon as the semantic information is available. Hence, an LRP was expected to develop on both go and no-go trials, because these are differentiated by the phonological information only subsequently. In the second version of the task, an LRP was expected for go trials only, because the go/no-go decision could be made based on the semantic information (which, by hypothesis, would be available early in time) while the selective response preparation could be initiated only if the phonological information became available (later on). This LRP pattern was indeed the one van Turennout et al. (1997) observed (see also Schmitt et al., 2000, for related findings). A similar pattern was obtained when replacing the semantic task by a syntactic task (grammatical gender decision, van Turennout et al., 1998). These findings are thus compatible with the notion that lemma access precedes word-form access. However, they are also in line with the idea that access to the two types of information proceeds in parallel, with the semantic (or syntactic) process terminating before the phonological process, as opposed to proceeding in two serially ordered steps. To explore this possibility, Abdel Rahman and Sommer (2000) contrasted an easy semantic task (decision on the natural size of a depicted animal, small vs. large) that was performed quickly with a difficult one (decision on the animal's diet, herbivore vs. carnivore) that was performed more slowly. When the easy semantic task was combined with the phonological task, the pattern obtained by van Turennout et al. and Schmitt et al. was replicated. By contrast, when the difficult semantic task was combined with the phonological task, an LRP on no-go trials was no longer obtained if the semantic decision was contingent on phonological properties, challenging the notion that semantic processing strictly precedes phonological processing. At first sight, these results seem to suggest that speakers can access the phonological form of a word without having retrieved its meaning. However, it could also be the case that some particular type of semantic information was only accessed because the decision task demanded its retrieval. Put differently, there might exist one processing stream that proceeds from the processing of a restricted set of semantic properties required for lexical access to the processing of phonological information in a serial way and a second processing stream that derives additional semantic properties running in parallel and operating independent from the phonological retrieval process. ${ }^{1}$

All these studies have established the LRP technique as a novel and fruitful approach for exploring lexicalization processes as they unfold in time. Still, it is debatable to which extent binary classification tasks resemble normal language production. Typically, a speaker processes semantic, syntactic, and phonological information in a highly automatic fashion and only a fraction of it becomes available to consciousness (see Levelt, 1989, p. 20ff). The LRP task, by contrast, requires participants to use this information for feeding a complex, voluntary, and metalinguistic decision. Given this difference, one cannot exclude the possibility that there are factors that contribute to the performance in the LRP paradigm that do not figure in normal speaking. One step towards a solution of this problem is provided in recent MEG studies of picture naming by Maeß, Friederici, Damian, Meyer, and Levelt (2002) and Levelt, Praamstra, Meyer, Helenius, and Salmelin (1998) in which dipole source analyses were used to localize the cortical areas that were active during successive stages of a regular picture-naming process. In the following, we present a novel technique based on ERP recordings that also resembles the normal production process rather closely, just like the study by Levelt et al. Our technique combines a delayed picture-naming task with a priming procedure and is very similar to chronometric procedures that have successfully been applied to the study of lexical access in language production (e.g., Peterson \& Savoy, 1998; Levelt et al., 1991). In fact, our approach can be seen as an "electrified" version of this approach. As we will see below, our approach forms the natural complement of LRP studies. LRP studies provide a 
detailed picture of the timing with which different types of information (semantic, phonological, and syntactic information) become available, but they do so at the price of explicitly forcing participants to retrieve these types of information. Our approach, by contrast, has a limited potential for addressing the time course issue, but it provides evidence as to what types of information become available automatically. Before describing the procedure in detail, some words on priming effects in ERP studies seem to be in place.

\section{Semantic and Phonological Priming Effects in ERP Studies}

In a typical priming study, a target word is preceded by a prime word or sentence fragment and the effect of the first word or fragment on the processing of the target word is assessed. In electrophysiological studies, semantic as well as phonologically related primes modulate the amplitude of the $\mathrm{N} 400$ with primed targets eliciting an attenuated N400 component.

\section{Semantic Influences on the N400}

Kutas and Hillyard $(1980,1983)$ showed that the ERPs to sentence final words forming a semantically incongruent completion elicited a negative shift peaking at around 400 msec relative to words providing a semantically congruent ending. More generally, the N400 amplitude is inversely related to the strength of meaning relation between a sentence and a critical open-class word. Parallel results have also been demonstrated for words embedded in lists and for word pairs (e.g., Brown \& Hagoort, 1993; Bentin, McCarthy, \& Wood, 1985). This difference in the N400 amplitude is referred to as the N400 priming effect and is also observed for auditorily presented words (e.g., Radeau, Besson, Fonteneau, \& Castro, 1998; Anderson \& Holcomb, 1995; Bentin, Kutas, \& Hillyard, 1995; Holcomb \& Neville, 1990) as well as for cross-modal presentation of prime and target (Holcomb \& Anderson, 1993).

\section{Phonological Influences on the N400}

Several studies showed that an N400 priming effect is also obtained with phonologically related primes. For example, when participants perform rhyme judgments on visually presented word pairs, unrelated stimuli elicit a more negative-going waveform than rhyming stimuli, peaking about $450 \mathrm{msec}$ after onset of the second word (Rugg, 1984a, 1984b). Similar effects are obtained in the auditory domain. For example, Praamstra and Stegeman (1993) observed an attenuated negativity about 300-600 msec after onset of the second word when it was preceded by a rhyming word as compared to a phonologically unrelated word. This effect was also observed if participants performed a lexical decision to the second stimulus in place of a rhyme judgment (see also Radeau et al., 1998). This is an important finding, because in lexical decisions no conscious attention needs to be directed to the target word's phonology. This finding thus suggests that the modulation of the N400 reflects phonological processes regularly involved when processing an auditory target word. Later research then revealed that the phonological N400 effect was sensitive to the time course with which phonological information becomes available in auditory word recognition (i.e., the effect starts earlier with word-initial overlap as opposed to word-final overlap, see Praamstra, Meyer, \& Levelt, 1994; see also van Petten, Coulson, Rubin, Plante, \& Parks, 1999, for related evidence with respect to the retrieval of semantic information).

In short, semantic and phonological effects from a prime word on the auditory N400 can be obtained even if conscious processing of the target word at either a semantic or a phonological level is not demanded. The paradigm to be described next makes use of this observation.

\section{The Experimental Paradigm}

The experimental paradigm combines a delayed picturenaming task with a priming procedure. Participants prepared the production of a picture name and vocally responded on receiving an articulation cue (“!!!”). On the experimental trials, they heard an auditory target word prior to receiving the cue. In this case, presentation of the articulation cue was further delayed until the offset of the auditory target. This target word was either semantically related to the prepared picture name, phonologically related, or unrelated. The trial scheme is illustrated in Figure 1.

The logic behind this procedure is closely related to an experimental technique that has repeatedly and successfully been applied in behavioral studies of language production (e.g., Peterson \& Savoy, 1998; Levelt et al., 1991). However, instead of having participants give a timed response on the target (e.g., lexical decision or reading aloud), we recorded ERPs to the target word. Thus, the paradigm can be considered as an "electrified" extension of an established language production paradigm. Preparation of the naming response should lead to activation of the corresponding semantic and phonological information in the lexical-conceptual system. We expected that this activation is reflected in the ERP response to the auditory target word; the ERP to the auditory target word should differ as a function of the relation between the self-generated prime and the target. In particular, the ERP waveform for a semantically or phonologically related prime picture should be attenuated when compared to an unrelated control, as in the abovementioned N400 priming studies. This prediction is not trivial. Rather, it presupposes that the self-generated verbal code is a similarly efficient 
Figure 1. Illustration of the experimental procedure.

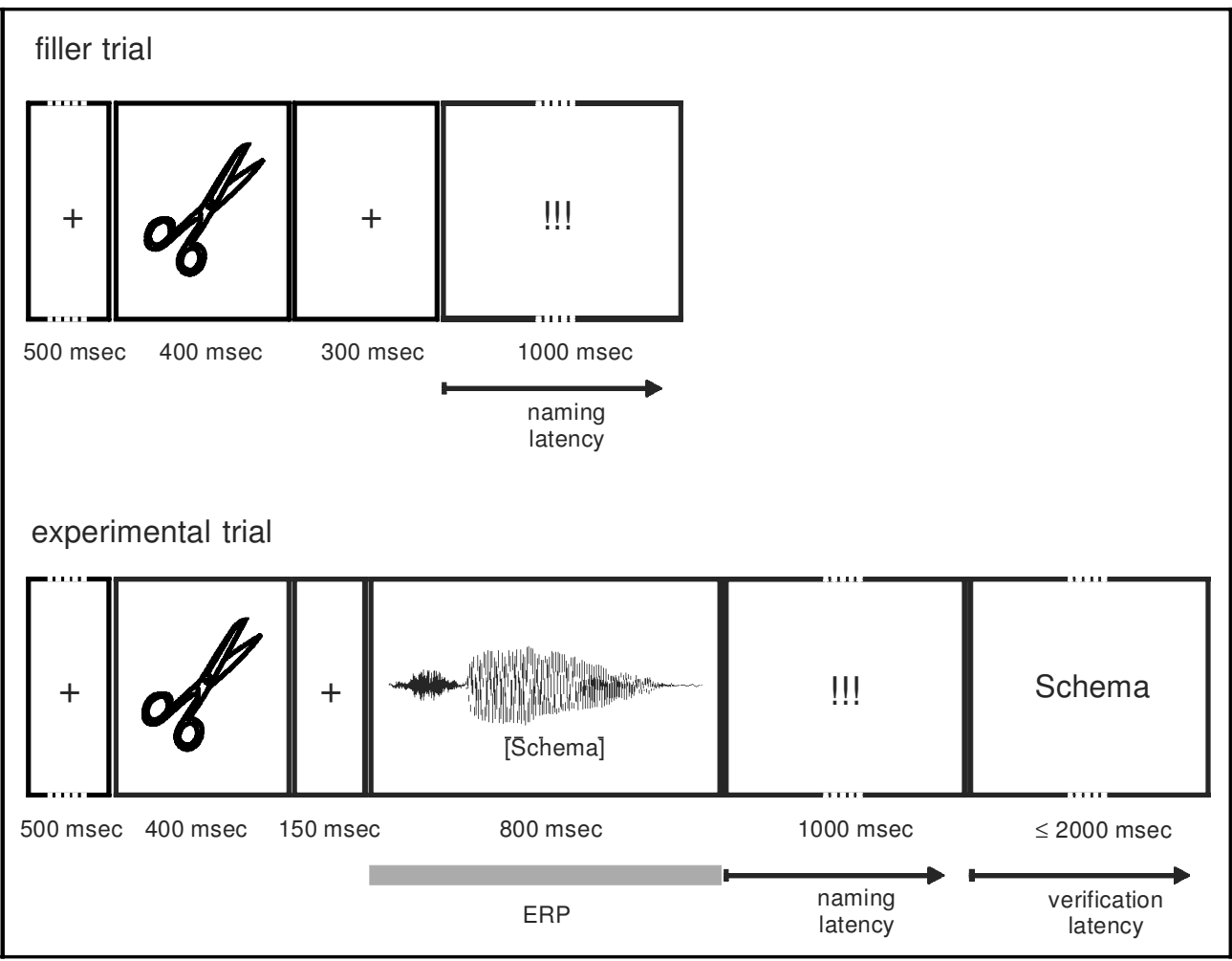

prime as an external stimulus presented in linguistic format (i.e., in the form of a written or spoken prime word). Thus, the ERPs should provide an indication of which codes (semantic and phonological codes) become automatically activated when planning to name a picture.

If such effects are obtained, the question arises whether they are the result of lexical retrieval processes required for object naming or the result of automatic spread of activation in the conceptual-lexical system. We tested these two possibilities by contrasting the lexical task (i.e., naming, Experiment 1) with a nonlexical task (Experiment 2). In the latter task, participants did not name the depicted objects but evaluated their natural size, relative to a fixed standard. While this task clearly requires conceptual-semantic processing of the picture stimulus, retrieval of its name is neither demanded nor functional. Any potential semantic and phonological ERP effects on the target words confined to the naming task could then be taken as an index of specific lexical retrieval processes. Note that any such dissociation of ERP effects between the naming task and the size-judgment task will also be informative with respect to competing models of lexical access in speech production. For example, in a fully cascaded model (cf., Humphreys, Riddoch, \& Quinlan, 1988) both tasks should yield the same results. This is the case because in such a model lexical information will always be activated as soon as the corresponding concept has become activated, even if the lexical information is irrelevant for the task at hand. By contrast, in serial-stage models, processing of conceptual-semantic information without activation of a phonological code is perfectly conceivable (cf., Levelt et al., 1991, 1999). We will return to this issue below, when discussing the ERP results.

We designed the experimental paradigm to ensure that participants (a) indeed implicitly generated the prime words-otherwise no lexical priming effect was to be expected, and (b) attentionally processed the target words - this seemed to be a prerequisite for the elicitation of a reliable N400 (e.g., Bentin et al., 1995; Chwilla, Brown, \& Hagoort, 1995; Brown \& Hagoort, 1993). To meet criterion (a), participants overtly produced the prepared word on all trials with some delay following a response cue (cf. Forster \& Chambers, 1973). To meet criterion (b), participants were additionally asked to decide whether a visual probe word presented at the end of the trial matched the auditory target word. To keep participants in a naming mode throughout the experimental session, the response cue delays were kept as short as possible, also in the experimental trials. In those trials, the response cue appeared $800 \mathrm{msec}$ after onset of the target words. This set the upper limit for the period during which the ERP could be measured. However, for all we know from the literature, this period should be long enough to detect any modulations of the ERP to the auditory target words, due to priming from the prepared picture name. Using this basic technique, the two experiments to be reported next explored the activation of semantic and phonological codes during lexical processing (delayed naming, Experiment 1) and 
Table 1. Mean Reaction Times (in Milliseconds) and Error Rates (in Percentage) in Delayed Naming and Probe Verification from Experiment 1

\begin{tabular}{lccccc}
\hline & \multicolumn{2}{c}{ Delayed Naming } & & \multicolumn{2}{c}{ Probe Verification } \\
\cline { 2 - 3 } Condition & $M$ & $\%$ & & $M$ & $\%$ \\
\hline sem-rel & $320(11)$ & $2.1(.8)$ & & $523(23)$ & $2.9(.7)$ \\
sem-unr & $321(11)$ & $2.5(.8)$ & $526(23)$ & $.8(.4)$ \\
pho-rel & $328(10)$ & $0.6(.3)$ & & $530(24)$ & $2.1(.5)$ \\
pho-unr & $321(10)$ & $1.0(.6)$ & & $519(19)$ & $1.9(.6)$ \\
filler & $383(12)$ & $0.9(.4)$ & & $-{ }^{\mathrm{a}}$ & $-{ }^{\mathrm{a}}$ \\
\hline
\end{tabular}

Standard errors are in parentheses.

sem-rel = prime picture and target word semantically related; sem-unr $=$ prime picture and target word semantically unrelated; pho-rel $=$ prime picture and target word phonologically related; pho-unr $=$ prime picture and target word phonologically unrelated; filler $=$ filler trials.

${ }^{a}$ No probes were presented in these trials.

conceptual processing (delayed size judgment, Experiment 2) of the prime picture.

\section{RESULTS AND DISCUSSION}

\section{Experiment 1}

\section{Behavioral Measures}

Table 1 displays reaction times and error rates for delayed naming and probe verification. The analysis of naming latencies for the four experimental conditions did not yield any reliable effects (most $F$ s $<1$, all $p s>.22)$. The analysis of error rates only revealed that more errors were made in the two semantic conditions than in the two phonological conditions, $F_{1}(1,19)=$ $5.14, p<.05 ; F_{2}(1,47)=8.02, p<.01$. Note however, that this difference concerns a comparison of naming performance with different probe words. Important for the issue at hand is the fact that the related and unrelated conditions (using the same probe words) did not differ in naming performance. In the analysis of probe verification latencies, there were no reliable effects (all $F \mathrm{~S}<1$ ). The analysis of the corresponding error rates showed that relatedness was reliable in the participant analysis, $F_{1}(1,19)=6.07, p<.05$, but not in the item analysis, $F_{2}(1,188)=2.43, p=.12$. A similar pattern held for the interaction of relatedness and type of relation, $F_{1}(1,19)=4.54, p=.05 ; F_{2}(1,188)=1.62$, $p=.20$.

In summary, there was only little effect of the experimental variables on the behavioral data. The only effect that could be confirmed in both the participant and the item analysis (more naming errors in semantic trials than in phonological trials) does not corrupt the specific comparisons of a related condition and its unrelated control.

\section{ERP Measures}

The grand average ERP waveforms at midline electrodes for the semantic conditions and the phonological conditions are displayed in Figure 2. In the case of semantically or phonologically related prime pictures, the ERPs to the target words were less negative going when compared to the unrelated control. Both effects were widely distributed. However, they differed with respect to their onset with the phonological effect starting
Figure 2. Grand average ERPs $(n=20)$ for the semantic conditions (left panel) and the phonological conditions (right panel) time-locked to the onset of the auditory target word from Experiment 1. Solid lines depict related conditions, and dashed lines depict unrelated conditions. In this and all following ERP figures, negative voltage is plotted up. For presentation purposes, the data were low-pass filtered with $10 \mathrm{~Hz}$. All statistical analyses were performed on the unfiltered data.

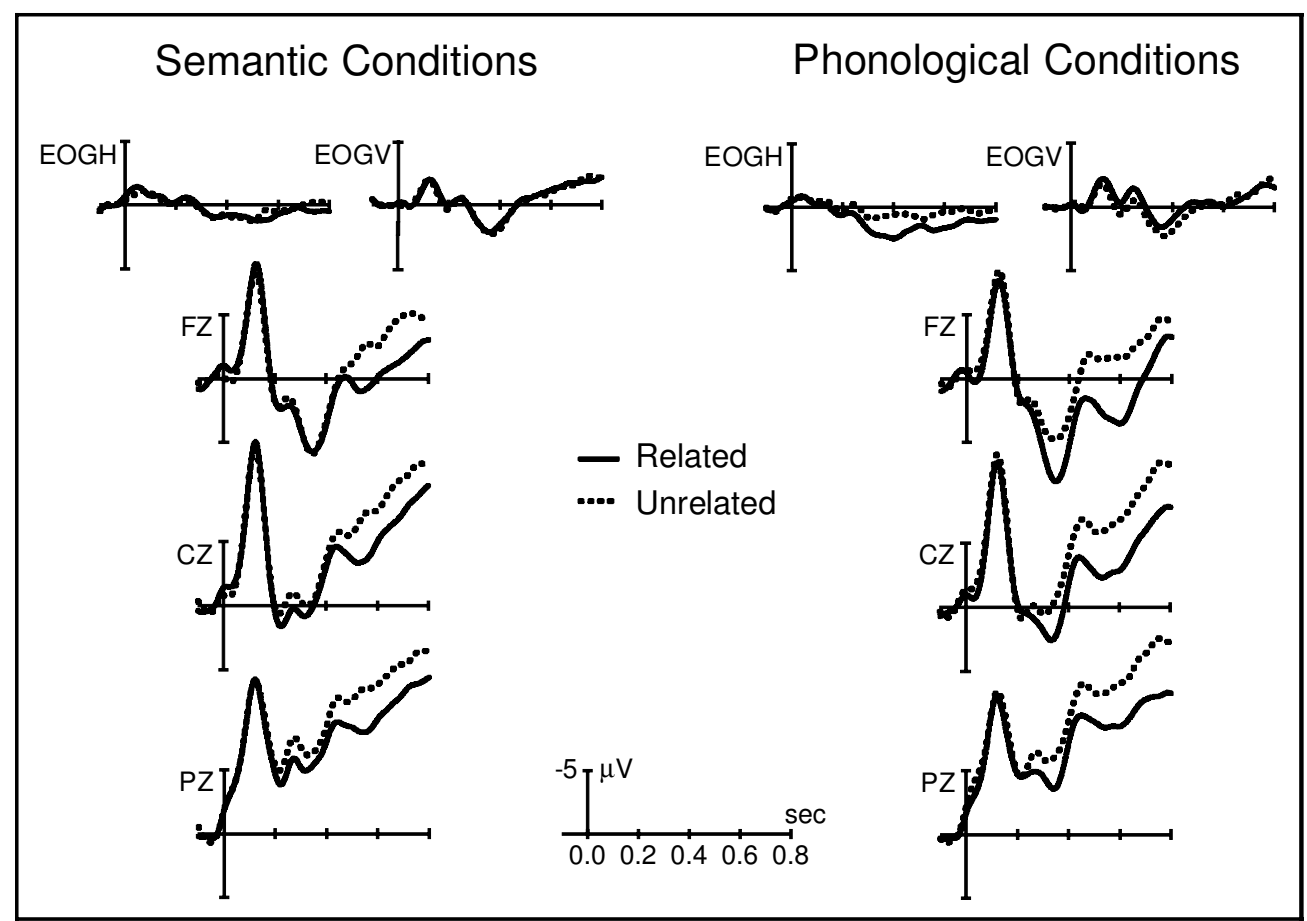


earlier than the semantic effect. We report analyses for two time windows (epoch 250-400 $\mathrm{msec}$ and epoch 400-800 msec).

Epoch 250-400 msec. SEMANTIC Conditions. There was neither a significant main effect of relatedness nor any reliable interaction involving this variable (all $p s>.19$ ). PHONOLOGICAL CONDITIONS. Relatedness was significant in the analysis of the lateral electrodes, $F(1,19)=8.85, p<.01$, with the unrelated condition yielding more negative amplitudes than the related condition. This effect was more pronounced over the right than the left hemisphere, $F(1,19)=32.79, p<.001$. Also, there was an interaction of relatedness, hemisphere, and region, $F(2,38)=11.28, p<.001$. Analyses per region of interest (ROI) revealed reliable relatedness effects for all but the left anterior and left central regions. The analysis of the midline electrodes largely confirmed this pattern. There was a significant relatedness effect, $F(1,19)=9.17$, $p<.001$, with the unrelated condition yielding a more negative ERP than the related condition. There was no interaction with electrode.

Epoch 400-800 msec. SEMANTIC CONDITIONS. Unrelated primes led to more negative amplitudes than related primes, yielding a significant effect of relatedness, $F(1,19)=35.29, p<.001$. This effect did not interact with either hemisphere or region $(p s>.15)$. Analysis of the midline electrodes confirmed this pattern. There was an effect of relatedness, $F(1,19)=40.39, p<.001$, that did not interact with electrode $(F<1)$. PHONOLOGICAL CONDITIONS. Unrelated primes led to more negative amplitudes than related primes, $F(1,19)=17.31$, $p<.001$. Although significant in both hemispheres, this effect was more pronounced over the right hemisphere, $F(1,19)=10.10, p<.01$. There was also an interaction of relatedness, hemisphere, and region, $F(2,38)=6.33$, $p<.01$. An analysis per ROI showed that the effect of relatedness was not significant in the left anterior region, and less pronounced in the left central region than in the remaining four regions. The analysis of the midline electrodes revealed a significant effect of relatedness, $F(1,19)=23.80, p<.001$. ERPs to unrelated words were more negative than ERPs to related words. There was no interaction with electrode.

\section{Discussion}

If the prepared picture name and the target word were semantically or phonologically related, the ERP to the target words tended less towards the negative as compared to the control condition. While the phonological effect was observed as early as $250 \mathrm{msec}$ after target onset, the semantic effect became visible only about 400 msec after target onset. Both effects had a broad topographic distribution and persisted until the end of the recorded epoch. While the semantic effect was about equally strong over both hemispheres, the phonological effect was most pronounced over midline and right electrode sites, as has also been observed in some previous studies (e.g., Rugg, 1984a, 1984b).

At first glance, the temporal sequence of the obtained effects-phonological effects starting earlier than semantic effects-appears somewhat unexpected, given the general assumption that processing during speech production proceeds from meaning to sound. Note, however, that our paradigm takes the brain's response to the auditory target word that has a certain relation to the prime (i.e., the generated picture name) as an index of lexical-conceptual activation induced by the production process. When taking into account the temporal characteristics of auditory word recognition, the obtained pattern makes much sense. An auditory word extends in time, over some hundred milliseconds. Importantly, the different types of lexical information it conveys may be recovered at different points in time. In the case of word-initial phonological overlap of prime and target, the phonological information becomes available as soon as the signal starts. By contrast, the semantic information may be recovered only later, after the initial phonological segments have been processed (e.g., Van Petten et al., 1999; Connolly \& Phillips, 1994). Hence, effects from phonological primes might surface at earlier points in time than effects from semantic primes, even if the time course of the production process runs opposite to this. On this account, one would predict that the onset of phonological priming effects depends on the position of the phonological segments shared between prime and target. With beginrelated word pairs the effect should start earlier than with end-related word pairs, just as is empirically observed (Jescheniak, Hahne, \& Schriefers, submitted; Praamstra et al., 1994).

Having established a procedure to tap the activation of semantic and phonological codes during the preparation of an overt naming response, the question arises whether the observed effects are indeed reflections of lexical retrieval processes during object naming. On an alternative account, they might be viewed as the result of some automatic spread of activation within the lexical-conceptual system. That is, any concept activated by the picture might automatically activate the corresponding lexical codes, regardless of whether the lexical code is needed for performing the task or not.

Experiment 2 sought to distinguish these two options. It used the same materials and the same priming procedure as Experiment 1. However, rather than performing a delayed picture-naming task, participants performed a delayed size-judgment task. In this task, they evaluated the natural size of the depicted object relative to a fixed standard. If performing this nonlexical task involves the activation of the semantic codes that led to the semantic ERP effect in Experiment 1, a similar semantic effect should be obtained. If it additionally involves the automatic activation of a phonological code, we should also replicate the phonological ERP effect. 
Note that phonological priming is reflected in the ERP irrespective of whether the task requires articulation or not (e.g., Rugg, 1984a, 1984b; Rugg \& Barrett, 1987). Thus, the absence of a phonological priming effect in Experiment 2 would imply that in the size-judgment task there is no activation of the picture's abstract phonological code.

\section{Experiment 2}

\section{Behavioral Measures}

Table 2 displays reaction times and error rates for size judgment and probe verification. The analysis of delayed size judgment latencies and error rates for the four experimental conditions did not reveal any reliable effects. In the analysis of the probe verification data, responses in the semantic conditions were slightly faster than responses in the phonological conditions but this effect was only reliable in the participant analysis, $F_{1}(1,27)=6.83, p<.05 ; F_{2}(1,188)=1.53, p=.22$. Likewise, the interaction of type of relation and relatedness was significant in the participant analysis and only marginally significant in the item analysis, $F_{1}(1,27)=$ $8.25, p<.01 ; F_{2}(1,188)=3.00, p=.09$. Simple comparisons showed that the difference between the semantically unrelated condition and the semantically related condition was reliable in the participant analysis but only marginally significant in the item analysis, $t_{1}(27)=3.22$, $p<.01 ; t_{2}(94)=1.87, p=.07$. The difference between the phonologically related condition and the phonologically unrelated condition, however, was not reliable, $t_{1}(27)=0.88, p=.38 ; t_{2}(94)=0.61, p=.54$. In the analyses of probe verification errors, no effect approached significance (all $F_{S}<1$ ).

In summary, condition effects on the behavioral data were weak. Although there were some tendencies in the

Table 2. Mean Reaction Times (in Milliseconds) and Error Rates (in Percentage) in Delayed Size Judgment and Probe Verification from Experiment 2

\begin{tabular}{lccccc}
\hline & \multicolumn{2}{c}{ Delayed Size Judgment } & & \multicolumn{2}{c}{ Probe Verification } \\
\cline { 2 - 3 } Condition & $M$ & $\%$ & & $M$ & $\%$ \\
\hline sem-rel & $342(14)$ & $2.7(.5)$ & & $592(22)$ & $3.4(.5)$ \\
sem-unr & $340(14)$ & $2.1(.5)$ & & $570(20)$ & $2.4(.7)$ \\
pho-rel & $348(14)$ & $1.3(.4)$ & & $589(21)$ & $2.4(.5)$ \\
pho-unr & $346(14)$ & $2.5(.7)$ & & $597(22)$ & $2.5(.6)$ \\
filler & $403(18)$ & $2.5(.5)$ & & $-{ }^{\text {a }}$ & $-^{-a}$ \\
\hline
\end{tabular}

Standard errors are in parentheses.

sem-rel = prime picture and target word semantically related; sem-unr $=$ prime picture and target word semantically unrelated; pho-rel $=$ prime picture and target word phonologically related; pho-unr $=$ prime picture and target word phonologically unrelated; filler $=$ filler trials.

${ }^{a}$ No probes were presented in these trials. probe verification latencies, they could not be confirmed in the item analyses. Most importantly, the size judgment data were not affected by our experimental manipulations, as had been the naming latencies in Experiment 1.

\section{ERP Measures}

The grand average ERP waveforms at midline electrodes for the semantic conditions and the phonological conditions are displayed in Figure 3. The pattern for the semantic conditions resembles the one observed in Experiment 1 . In the case of a related prime picture, the ERPs to the target words tended less towards the negative when compared to the unrelated control. For the phonological conditions, by contrast, no effect was obtained.

Epoch 250-400 msec. SEMANTIC CONDITIONs. There was no significant effect of relatedness and no interaction involving this variable. PHONOLOGICAL CONDITIONS. There was no significant effect of relatedness and no interaction involving this variable.

Epoch 400-800 msec. SEMANTIC CONDITIONS. Relatedness was significant in the analysis of the lateral electrodes, $F(1,27)=7.98, p<.01$, and in the analysis of the midline electrodes, $F(1,27)=7.48, p<.05$, with less negativegoing waveforms in the related condition than in the unrelated condition. Relatedness did not interact with any of the topographic variables. PHONOLOGICAL CONDITIONS. There was no significant effect of relatedness for the main effect and all interaction $F \mathrm{~s}<1$.

\section{Discussion}

The behavioral measures demonstrate that participants had no difficulty in evaluating the natural size of the depicted objects (error rates less than 2.5\% and delayed response latencies below $400 \mathrm{msec}$ ). This high-level performance resembles the one from Experiment 1, involving object naming (less than 1.5\% errors, with slightly faster response latencies). As to the ERP data, a semantic effect was again obtained, with the ERP waveforms being attenuated after semantically related picture primes. This effect occurred in the same time window and exhibited a broad topographic distribution, as it had in Experiment 1 (see Figure 4). The presence of the semantic effect validates that the size judgment task is indeed sensitive to priming. Unlike in Experiment 1, however, there was no indication of a phonological ERP effect in this experiment (see Figure 5 ). ${ }^{2}$ This difference suggests that the activation of a phonological code is confined to the lexical task. It also suggests that the activation of a conceptual representation by a picture stimulus does not automatically lead to substantial (i.e., measurable) activation of a phonological code, if such activation is not demanded by the task.

In this context, it is also interesting to note that the semantic effect in the present experiment (size 
Figure 3. Grand average ERPs $(n=28)$ for the semantic conditions (left panel) and the phonological conditions (right panel) time-locked to the onset of the auditory target word from Experiment 2. Solid lines depict related conditions, and dashed lines depict unrelated conditions.

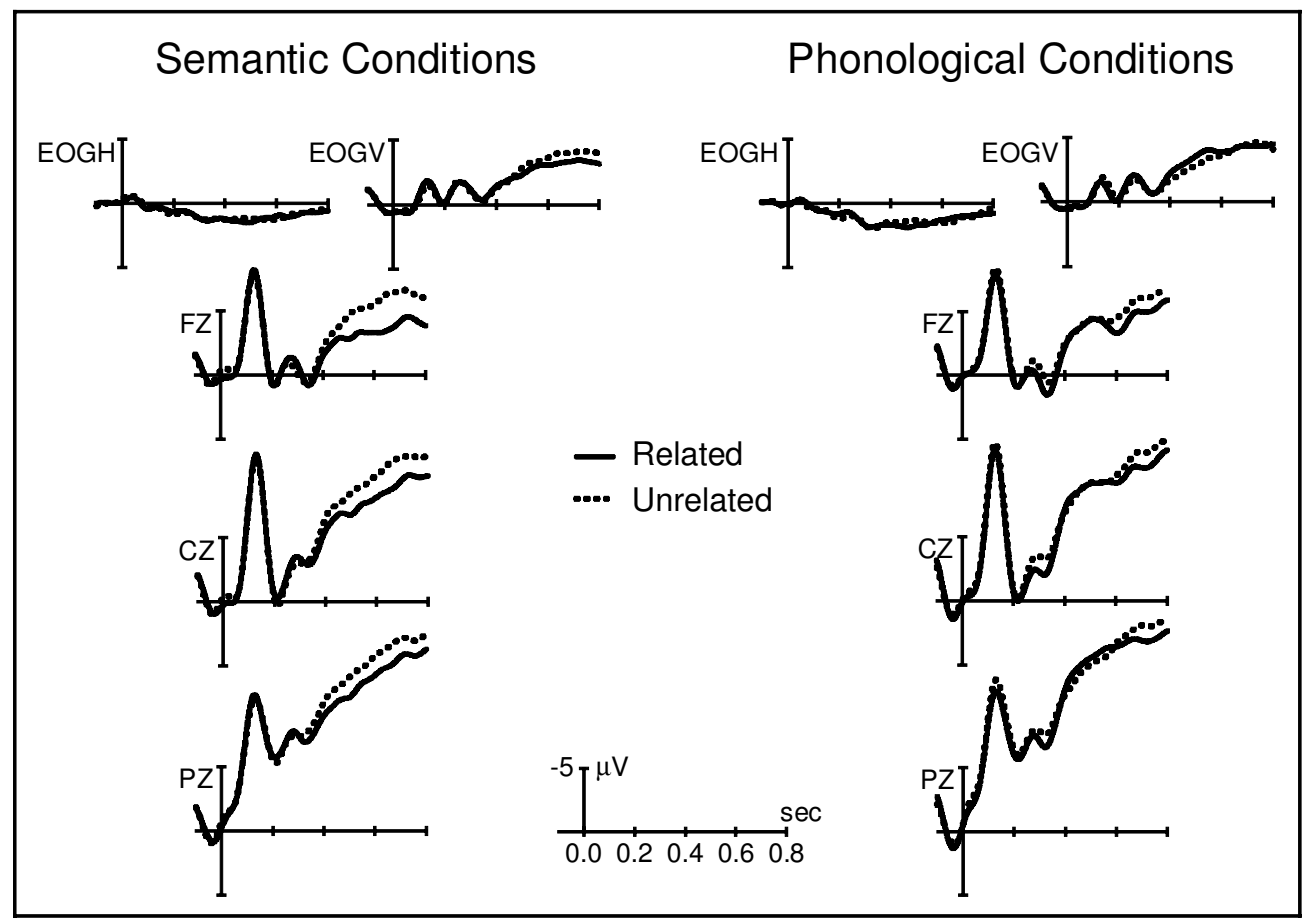

judgment) descriptively shows a slight topographic shift towards anterior electrode sites, while in Experiment 1 (picture naming) the frontal and occipital effects were of about equal size. A related observation has been reported by Ganis, Kutas, and Sereno (1996). In their study, prime sentences were followed by either a word or a picture as the target. The targets were either semantically congruous or incongruous with the prime. The authors observed that the N400 effect at posterior sites was larger for a semantic relation between two
Figure 4. Difference waves (unrelated-related) for the semantic conditions in Experiment 1 (solid line) and in Experiment 2 (dashed line) time-locked to the onset of the auditory target word.

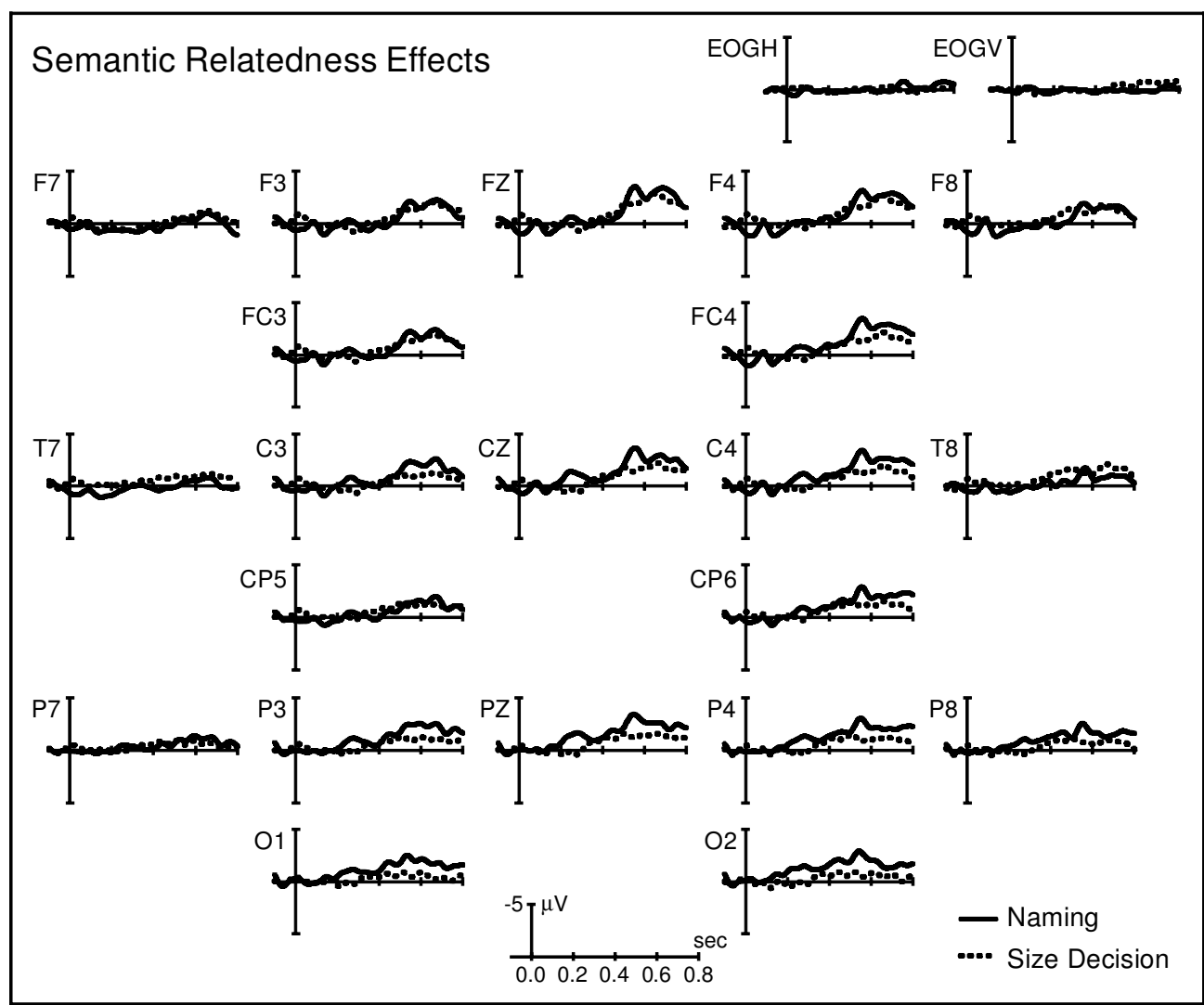


Figure 5. Difference waves (unrelated-related) for the phonological conditions in Experiment 1 (solid line) and in Experiment 2 (dashed line) time-locked to the onset of the auditory target word.

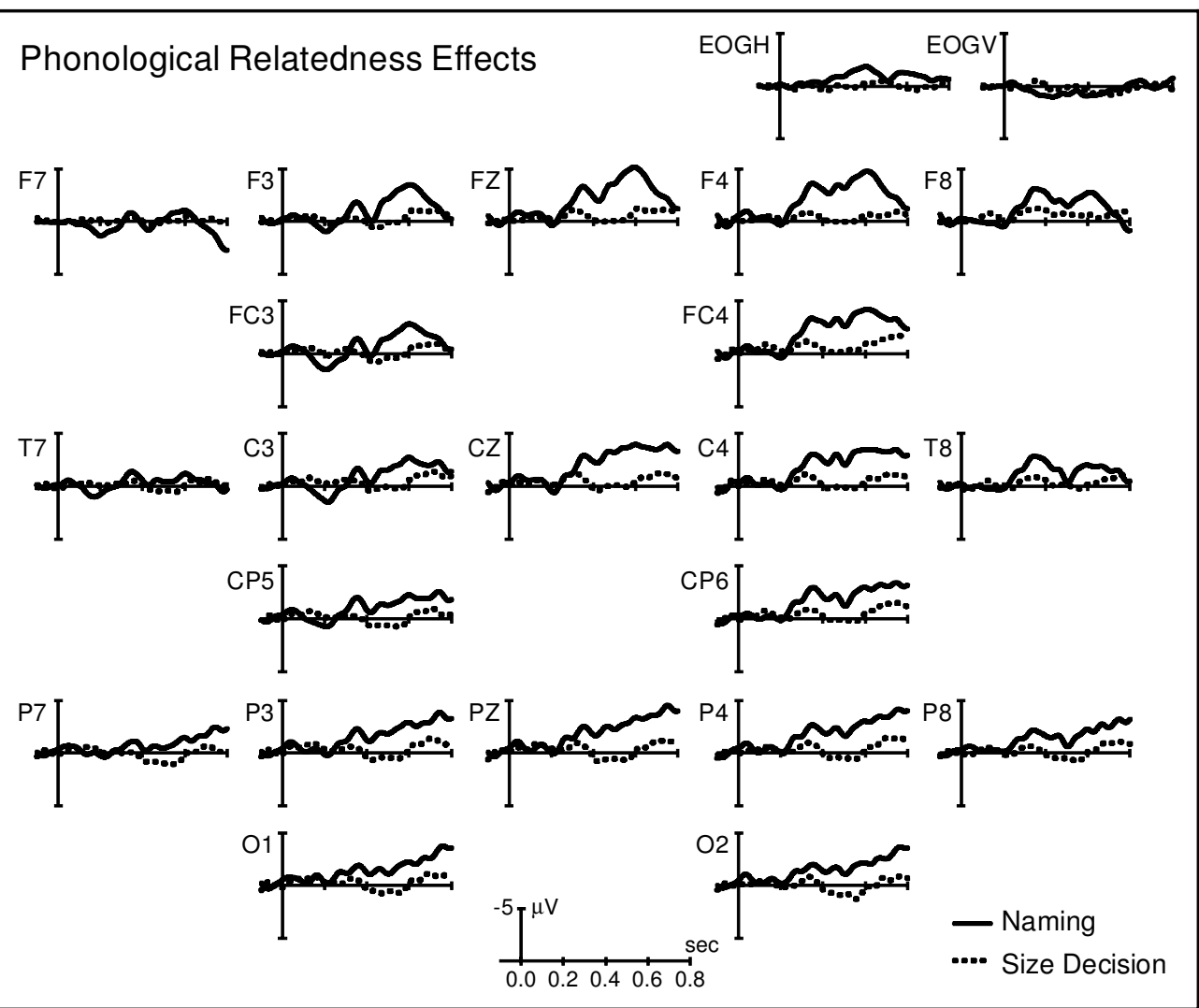

linguistic elements than for a semantic relation between a linguistic element and a picture. If one relates this observation by Ganis et al. to the difference of semantic effects in the present experiments, this could be taken as additional support that in Experiment 1 the pictures

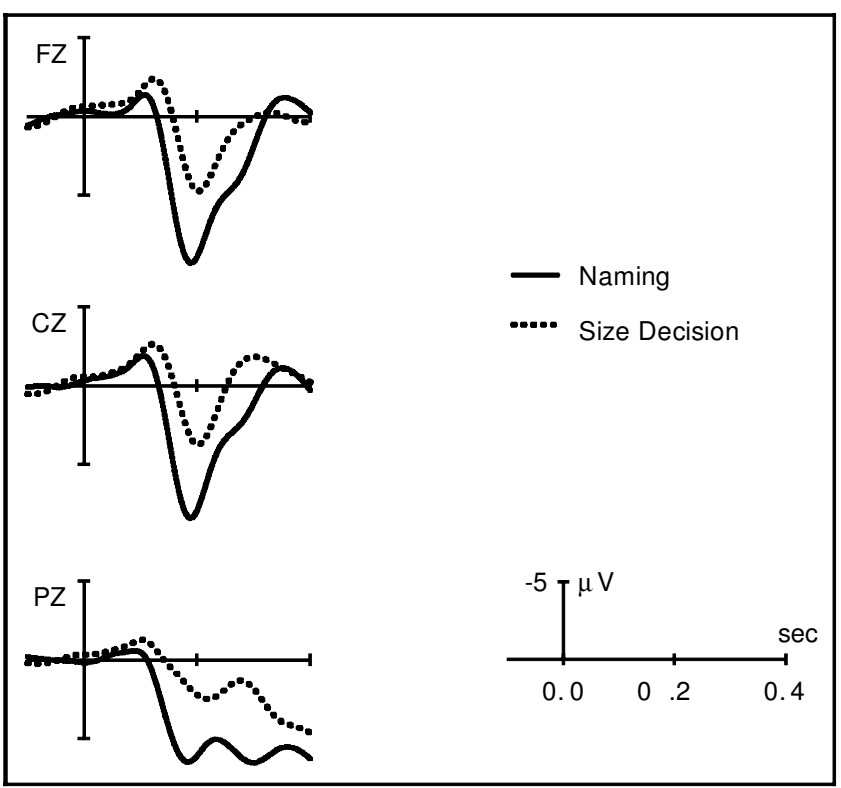

Figure 6. Grand average ERPs for the naming task in Experiment 1 (solid line) and the size-judgment task in Experiment 2 (dashed line) time-locked to the onset of the prime pictures. were in fact lexically coded and this code served as the prime, while in Experiment 2 this was not the case. Although admittedly speculative, this interpretation perfectly fits with the fact that we find an effect of phonological relatedness in Experiment 1 but not in Experiment 2. A definite conclusion on this issue, however, would require one to explore task-dependent topographic differences in the semantic effect in a within-participant design, as had been done in the study by Ganis et al.

Our interpretation that the task demands in the two experiments affected the processing of the picture is further supported by an analysis of the ERPs to the prime pictures (see Figure 6). These analyses revealed a more pronounced N2 in the naming task (Experiment 1) than in the size decision task (Experiment 2). ${ }^{3}$ Although this between-participant comparison needs to be treated with some caution and the functional significance of the effect is as yet not clear, it suggests that the pictures were indeed processed differently in Experiments 1 and 2 , in line with our assumptions.

\section{GENERAL DISCUSSION}

The ERP data showed clear semantic effects in both the naming task (Experiment 1) and the size-judgment task (Experiment 2). This suggests that in both tasks a semantic code is activated that can be tapped by semantically related target words. The activation of this code is 
presumably an automatic process as its conscious processing was not required for performing either of the tasks. This contrasts with the LRP technique discussed in the introduction (Schmitt et al., 2000; Abdel Rahman \& Sommer, 2000; van Turennout et al., 1997) in which a particular type of semantic information (e.g., animacy or diet) had to be processed such that it could feed the required conscious decision.

Another issue concerns the locus within the conceptual-lexical system at which the obtained semantic effects arise. While some authors argue for the existence of two levels of meaning representation (i.e., a prelinguistic semantic-conceptual level and a lexical-semantic level; see Bierwisch \& Schreuder, 1992; see also Hart \& Gordon, 1992, for evidence from a patient study), other authors maintain that semantic information is exclusively represented at a nonlexical, conceptual level (e.g., Levelt et al., 1999). Hence, the presence of the semantic effect in the naming as well as in the sizejudgment task is compatible with both its interpretation as the reflection of conceptual-semantic activation and its interpretation as the reflection of lexical-semantic (i.e., lemma) activation. The present data do not allow to distinguish between these two options, ${ }^{4}$ although some aspects of the present results at least suggest a difference in the semantic processing of pictures prepared for naming versus a nonverbal response. However, they do show that the activation of a semantic code does not automatically lead to a substantial activation of a phonological code, in line with previous behavioral findings (e.g., Jescheniak, Schriefers, \& Hantsch, 2001; LaHeij \& Bloem, 1999; Levelt et al., 1991). This argues against any models with unconstrained cascading of activation from semantic representations (be it conceptual or lexical) to phonological representations. Rather, phonological codes appear to be activated to a substantial degree only when they are required for generating the eventual response, that is, when naming a depicted object.

In summary, the experimental procedure described in this article provides a novel and sensitive ERP technique for exploring semantic and phonological processing in speech planning, supplementing the LRP approach. The LRP approach requires participants to explicitly and consciously extract semantic and phonological information, while the present approach taps its automatic activation. On the other hand, the LRP approach reveals important information on the timing of the different processing stages while the present approach has clear limitations in this respect. Thus, the two approaches can be seen as complementing each other. The fact that the present approach allows us to tap the type of codes that become automatically activated in language production opens up the possibility to apply this paradigm in future research to address other important issues in current language production research that are not easily addressed with existing procedures. One of these issues concerns the question of whether the phonological codes of semantic competitors of a to-be-produced word become automatically activated.

\section{METHODS}

\section{Participants}

Forty-eight healthy participants (21 men and 27 women, with a mean age of 23.8 years, ranging from 19 to 31 years) from the participant pool of the Max Planck Institute of Cognitive Neuroscience were tested in the experiments, 20 in Experiment 1 and 28 in Experiment 2. ${ }^{5}$ Most of them were students from the University of Leipzig and had participated in several unrelated ERP experiments previously. All of them were right-handed, native speakers of German, with no known hearing deficit, and normal or corrected-to-normal vision. They were paid for their participation (DM 13 per hour).

\section{Stimuli}

Our study included pictures of 48 simple objects that all had one unambiguous name (see Figure 7 for examples). We selected the materials such that the size-judgment task to be used in Experiment 2 required retrieval of detailed conceptual information and could not be solved by superficial perceptual analysis of the stimulus. For each object, two target words were selected. One of them belonged to the same semantic category as the object name (e.g., "Beil" [hatchet], if the picture name was "Schere" [scissors], semantically related condition), and the other minimally shared the initial consonant-vowel segments with the object name (e.g., "Schema" [scheme], phonologically related condition). Unrelated control conditions against which

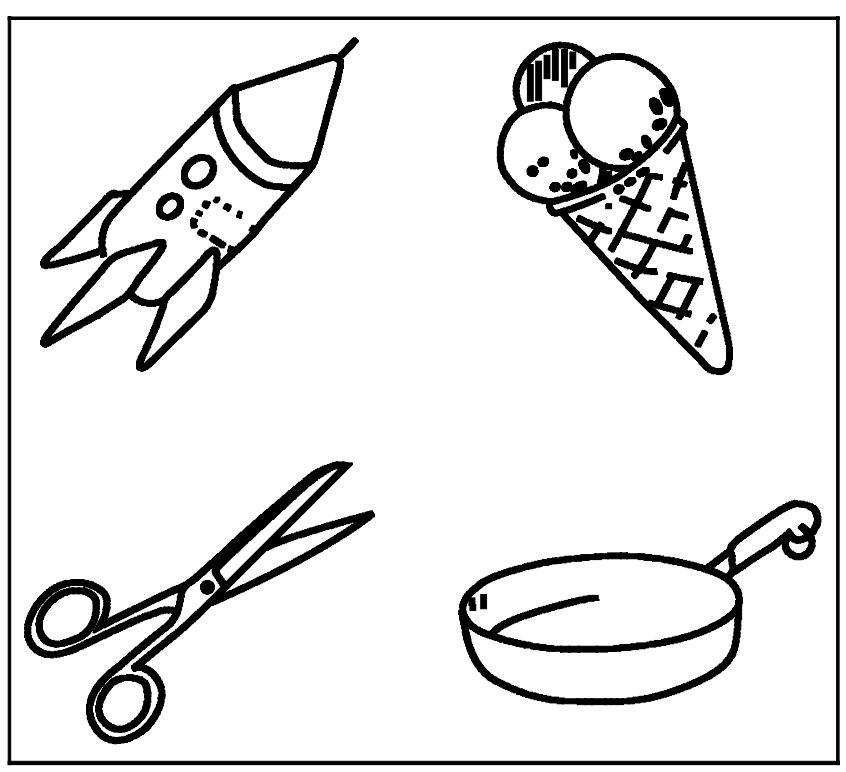

Figure 7. Illustration of the picture stimuli used in Experiments 1 and 2 . 
any specific effect of a semantically related or a phonologically related prime picture could be evaluated were created by reassigning the related prime pictures from the respective conditions to the target words. This way, we ensured that no incidental differences across prime picture sets would contribute to the observed effects. ${ }^{6}$ The semantic relatedness of semantically related stimuli and the semantic unrelatedness of all other stimuli was verified in an off-line norming task (with $N=40$ participants). Mean relatedness ratings on a 7-point scale (with 1 to be used for "words that are unrelated in meaning" and 7 to be used for "words that are highly related in meaning") were $5.36(S D=1.11), 1.54(S D=.51), 1.44$ $(S D=.50)$, and $1.35(S D=.45)$ for the semantically related condition, the semantically unrelated condition, and the two phonological conditions, respectively.

Prime pictures were scaled to a size of approximately $50 \times 50 \mathrm{~mm}$. Auditory target words were spoken by a woman native speaker of German, digitized at a sampling rate of $22 \mathrm{kHz}$, and further processed with sound editing software. In addition to these experimental materials, a set of nine pictures along with appropriate target words was selected to be used in practice trials.

Eight different versions of the experiment were created. In a first step, the 48 target words for the semantic conditions and the 48 target words for the phonological conditions were divided into two subsets containing 24 words each. Two parallel raw lists were then created such that each target word appeared only once in each list, but contributed-across lists-to both experimental conditions (related vs. unrelated). In half of the experimental trials-equally distributed across experimental conditions-positive probes were paired with the auditory target word, and in the other half negative probes were paired with the auditory target word. In each of the two raw lists, each prime picture was also presented twice in pure naming trials to ensure that participants would actively engage in preparing the naming response (in Experiment 1) or the size judgment (in Experiment 2) from the onset of the prime picture.

Each of the two raw lists was then divided into four experimental blocks such that in each block each prime picture appeared exactly once. Across subsets of prime pictures, the assignment of the four conditions to the four blocks was counterbalanced. In each block, half of the trials were filler trials and half were experimental trials with each of the four experimental conditions appearing exactly six times. For each block, two different random sequences were created. Each participant received all four blocks of one raw version. For half of the participants, the sequence of the four blocks was reversed.

\section{Procedure}

Participants were seated in a dimly lit soundproofed booth. In Experiment 1, they performed a delayed object-naming task, and in Experiment 2, they performed a delayed size-judgment task. In the latter task, participants decided whether a typical instance of the object fits into a box (about $26 \times 18.5 \times 12 \mathrm{~cm}$ ) that was placed in front of them. Participants were instructed to prepare their responses while the prime picture was presented and to initiate the overt (vocal or pushbutton) response as quickly as possible when a response cue ("!!!") appeared on the CRT. They were instructed to additionally listen to the auditory word appearing occasionally so they could later decide whether it matched a visual probe word presented after they had given the delayed response, by pressing a push-button. Participants pressed the left button labeled "yes" if auditory and visual word matched and the right button labeled "no" if otherwise. Participants were asked to move as little as possible during the presentation of the fixation signal and the pictures.

The box used for the size-judgment task in Experiment 2 was selected such that half of the objects could be placed inside the box while the other half could not, according to our intuition. Because our intuition need not match each individual participant's decision for each object without rendering this participant's decision incorrect, we adopted the following criterion for identifying errors. If a participant had given the same response during all four presentations of a particular picture in the main experiment, all responses were considered correct. If there was one deviant response, this single response was coded as erroneous. However, if there were two positive and two negative responses, all four responses were coded as erroneous. These responses were discarded from the analyses of reaction times and ERPs.

Before the experiment started, participants studied written instructions. The delayed naming (or judgment) task was introduced as the participants' primary task that they were instructed to perform with maximal speed and accuracy. For the subsequent probe verification task, accuracy rather than speed was emphasized. Participants also studied a booklet containing all picture stimuli used in the experiment. In the booklet used for Experiment 1, a name was printed next to each picture. Participants were instructed to use only these words for naming the objects. In the booklet used for Experiment 2, the object names were removed from the booklets. This allowed us to familiarize participants with the pictorial materials without encouraging implicit naming during the experiment. Participants were instructed to evaluate for each individual picture whether a typical instance of that object fits into the box while studying the booklet. They were asked to stick to their decision throughout the whole experiment. Next, participants were trained on the delayed response task with each experimental picture and each practice picture being presented once. In a second step, auditory target words and visual probe words 
additionally appeared in half of the trials. During this phase, only practice pictures and words were presented in another 36 practice trials. Then the main experiment started. After half of the trials, there was a short pause. Each of the two parts of the main experiment started with five warm-up trials containing practice pictures and words.

Prime pictures and probe words were presented centered on a CRT in light gray on a black background. Viewing distance was about $100 \mathrm{~cm}$. The auditory target words were presented via loudspeakers adjusted to a comfortable listening volume. Each trial started with a fixation signal ("+") presented for $500 \mathrm{msec}$. It was replaced by the prime picture displayed for $400 \mathrm{msec}$. At the offset of the prime picture, the fixation signal reappeared. In the filler trials, the fixation signal remained visible for $300 \mathrm{msec}$, followed by the response cue presented for $1 \mathrm{sec}$. Two seconds later, the next trial began. In the experimental trials, the fixation signal remained visible for $950 \mathrm{msec}$, and was then replaced by the response cue presented for $1 \mathrm{sec}$. The auditory target word started $150 \mathrm{msec}$ after offset of the prime picture. The visual probe directly followed the response cue and remained on the screen until the participant pressed a button to indicate his/her probe verification response or, if no response was given, until 2 sec had elapsed. After two more seconds, the next trial began. Naming latencies were measured from the onset of the response cue with a microphone feeding a voice-key connected to the computer. Shorter cue delays were used in the experimental trials to reduce the chance that participants, while anticipating cue onset, would initiate articulation too early, thus spoiling some larger proportion of these trials. Observations were discarded and an error coded if (a) the picture was named other than expected, (b) no response was given within $1 \mathrm{sec}$, or (c) a response was initiated before the onset of the response cue. Reaction times on trials in which the voice-key malfunctioned but the participant responded correctly were discarded, without coding an error. Size decision latencies were also measured from the onset of the response cue. Errors in this task were identified based on each participant's response consistency according to the procedure described above. In the experimental trials, probe verification latencies were additionally measured, from the onset of the visual probe. For these, an error was coded whenever the latency exceeded 2 sec or participants responded incorrectly.

\section{EEG Recording}

The EEG was recorded monopolarly with $21 \mathrm{Ag} / \mathrm{AgCl}$ electrodes mounted in an elastic cap at the following positions: F7, F3, FZ, F4, F8, FC3, FC4, T7, C3, CZ, C4, T8, CP5, СР6, P7, P3, PZ, P4, P8, O1, and O2, referenced to the left mastoid. ${ }^{7}$ The horizontal electro- oculogram was recorded bipolarly from electrodes placed at the outer canthus of each eye, and the vertical electrooculogram was recorded bipolarly by electrodes placed above and below the right eye. Electrode impedance was kept below $5 \mathrm{k}$. . Electrical activity was amplified within a band pass from DC to $40 \mathrm{~Hz}$. The EEG was digitized with a sampling rate of $250 \mathrm{~Hz}$. ERPs were computed from the onset of the target word for a duration of $800 \mathrm{msec}$ (i.e., to the onset of the response cue) relative to a 100-msec prestimulus baseline separately for each of the four experimental conditions.

\section{Data Analyses}

For each participant, mean amplitudes were computed for each electrode and each experimental condition, averaging over artifact-free trials in which participants had given a correct response. Trials, on which a deviant naming response (in Experiment 1) or a deviant size decision (in Experiment 2) had been given, in which the response had been initiated before the onset of the response cue or with a latency exceeding $1 \mathrm{sec}$ were discarded from the analyses. Based on these criteria, a total of $7.8 \%$ of all trials was discarded in Experiment 1, $6.4 \%$ because of EEG artifacts, and $1.4 \%$ because of erroneous naming responses. In Experiment 2, a total of $9.1 \%$ of all trials was discarded, $7.3 \%$ because of EEG artifacts, and $1.8 \%$ because of erroneous size judgments. The percentages of excluded trials were distributed about equally across experimental conditions.

Based on a visual inspection of the grand average waveforms, we selected two time windows for analyses, an early time window ranging from 250 to $400 \mathrm{msec}$, and a late time window ranging from 400 to $800 \mathrm{msec}$. Analyses of variance (ANOVAs) were carried out for each time window separately on the semantic conditions and the phonological conditions. For the analysis of the lateral electrodes, six ROIs containing three electrodes each were defined: left anterior (F7, F3, FC3), left central (T7, C3, CP5), left posterior (P7, P3, O1), right anterior (F4, F8, FC4), right central (C4, T8, CP6), and right posterior $(\mathrm{P} 4, \mathrm{P} 8, \mathrm{O} 2)$. The analysis of these electrodes included relatedness (related vs. unrelated), hemisphere (left vs. right), region (anterior vs. central vs. posterior) as completely crossed within-participant variables. The analysis of the midline electrodes included relatedness and electrode (FZ vs. CZ vs. PZ) as completely crossed within-participant variables. Where appropriate, the Greenhouse-Geisser correction for inhomogeneity of variance was applied. We report uncorrected degrees of freedom and probability levels following correction.

For each experiment, delayed naming or conceptual decision latencies, probe verification latencies, and the respective error rates were submitted to ANOVAs with the crossed variables type of relation (semantic vs. 
phonological) and relatedness (related vs. unrelated). Latencies deviating more than $2 S D$ s from a participant's and an item's mean were discarded as outliers. There were two analyses. In one analysis, participants were treated as random variable $\left(F_{1}\right)$, and in a second analysis, items were treated as random variable $\left(F_{2}\right)$.

\section{Acknowledgments}

This work was supported by grant DFG Je229/4-1 and a Heisenberg fellowship from the German Research Council awarded to the first author. We thank Anja Hahne for her advice during the course of the project. Thanks also to Ina Koch, Cornelia Schmidt, and Valentin Wagner for invaluable help in data collection.

Reprint requests should be sent to Jörg D. Jescheniak, Max Planck Institute of Cognitive Neuroscience, P. O. Box 500355, D-04303 Leipzig, Germany, or via e-mail: jeschen@cns.mpg.de.

\section{Notes}

1. For example, according to Levelt et al. (1999), holistic conceptual representations are mapped onto lexical representations during lexical access or, as Roelofs (1992) put it, "only a very limited and shallow part of the conceptual structure interacts with syntax. Conceptual complexity remains outside the message"' (p. 26).

2. Upon visual inspection, however, one might wonder whether there is a late-developing effect over medial electrode sites for the phonological conditions. However, this descriptive difference was not reliable. When performing analyses on successive $50-\mathrm{msec}$ time windows, there was neither reliable effect of phonological relatedness nor any interaction involving this variable in any of the time windows. All there was is a trend $(p=.08)$ between 700 and $750 \mathrm{msec}$ for lateral electrode sites, which, however, was not replicated for the midline electrodes $(p>.10)$. Likewise, an analysis on the time window ranging from 580 to $800 \mathrm{msec}$ did not reveal any such effects (all Fs $<1$ ). Possibly, the minor descriptive difference was caused by individual participants who implicitly named the objects on a few trials, although it was not required by the task.

3. An analysis in the $170-220-\mathrm{msec}$ time window revealed a highly significant effect of task for both lateral electrodes, $F(1,46)=17.54, p<.001$, and midline electrodes, $F(1,46)=$ 18.24, $p<.001$.

4. These options are generally difficult to disentangle given that some models maintain that a word's lemma will always be activated when the concept has been activated (e.g., Levelt et al., 1999).

5. More participants were tested in Experiment 2 as to exclude the possibility that potentially attenuated ERP effects in this experiment could be overlooked due to insufficient statistical power.

6. Half of the materials were taken from Jescheniak et al. (2001). A complete list of the materials is available from the first author.

7. Additional data analyses were performed after re-referencing the EEG off-line to the average of the left and the right mastoid. These analyses yielded highly comparable results. In particular, the hemispheric asymmetry of the phonological effect in Experiment 1 was still obtained, suggesting that it cannot be attributed to our particular choice of reference electrode.

\section{REFERENCES}

Abdel Rahman, R., \& Sommer, W. (2000). Parallel access to semantics and phonology in object recognition? Journal of Cognitive Neuroscience, 12, Supplement, 124.

Anderson, J. E., \& Holcomb, P. J. (1995). Auditory and visual semantic priming using different stimulus onset asynchronies: An event-related brain potential study. Psychophysiology, 32, 177-190.

Bentin, S., Kutas, M., \& Hillyard, S. (1995). Semantic processing and memory for attended and unattended words in dichotic listening: Behavioral and electrophysiological evidence. Journal of Experimental Psychology. Human Perception and Performance, 21, 54-67.

Bentin, S., McCarthy, G., \& Wood, C. C. (1985). Event-related potentials, lexical decision, and semantic priming. Electroencephalography and Clinical Neurophysiology, 60, 343-355.

Bierwisch, M., \& Schreuder, R. (1992). From concepts to lexical items. Cognition, 42, 23-60.

Brooker, B. H., \& Donald, M. W. (1980). Contribution of speech musculature to apparent EEG asymmetries prior to vocalization. Brain and Language, 9, 226-245.

Brown, C. M., \& Hagoort, P. (1993). The processing nature of the N400: Evidence from masked priming. Journal of Cognitive Neuroscience, 5, 34-44.

Chwilla, D. J., Brown, C. M., \& Hagoort, P. (1995). The N400 as a function of the level of processing. Psychophysiology, 32, $274-285$.

Coles, M. G. H., Gratton, G., \& Donchin, E. (1988). Detecting early communication: Using measures of movement-related potentials to illuminate human information processing. Biological Psychology, 26, 69-89.

Connolly, J. F., \& Phillips, N. A. (1994). Event-related potential components reflect phonological and semantic processing of the terminal word of spoken sentences. Journal of Cognitive Neuroscience, 6, 256-266.

de Jong, R., Wierda, M., Mulder, G., \& Mulder, L. J. M. (1988). Use of partial information in responding. Journal of Experimental Psychology. Human Perception and Performance, 14, 682-692.

Dell, G. S., \& O'Seaghdha, P. G. (1992). Stages of lexical access in language production. Cognition, 42, 287-314.

Forster, K. I., \& Chambers, S. (1973). Lexical access and naming time. Journal of Verbal Learning and Verbal Behavior, 12, 627-635.

Ganis, G., Kutas, M., \& Sereno, M. I. (1996). The search for "common sense": An electrophysiological study of the comprehension of words and pictures in reading. Journal of Cognitive Neuroscience, 8, 89-106.

Garrett, M. F. (1988). Processes in language production. In F. J. Newmeyer (Ed.), The Cambridge survey of linguistics, Language: Psychological and biological aspects (vol. 3, pp. 69-96). Cambridge: Harvard University Press.

Gratton, G., Coles, M. G. H., Sirevaag, E., Eriksen, C. W., \& Donchin, E. (1988). Pre- and poststimulus activation of response channels: A psychophysiological analysis. Journal of Experimental Psychology. Human Perception and Performance, 14, 331-506.

Hart, J., Jr., \& Gordon, B. (1992). Neural subsystems for object knowledge. Nature, 359, 60-64.

Holcomb, P. J., \& Anderson, J. E. (1993). Cross-modal semantic priming: A time-course analysis using event-related brain potentials. Language and Cognitive Processes, 8, 379-411.

Holcomb, P. J., \& Neville, H. J. (1990). Semantic priming in visual and auditory lexical decision. A between modality comparison. Language and Cognitive Processes, 5, $281-312$ 
Humphreys, G. W., Riddoch, M. J., \& Quinlan, P. T. (1988). Cascade processes in picture identification. Cognitive Neuropsychology, 5, 67-103.

Jescheniak, J. D., Hahne, A., \& Schriefers, H. (submitted). Information flow in the mental lexican during speech planning: Evidence from event-related brain potentials.

Jescheniak, J. D., Schriefers, H., \& Hantsch, A. (2001). Semantic and phonological activation in noun and pronoun production. Journal of Experimental Psychology. Learning, Memory, and Cognition, 27, 1058-1078.

Kutas, M., \& Hillyard, S. A. (1980). Reading senseless sentences: Brain potentials reflect semantic incongruity. Science, 207, 203-205.

Kutas, M., \& Hillyard, S. A. (1983). Event-related potentials to grammatical errors and semantic anomalies. Memory and Cognition, 11, 539-550.

LaHeij, W., \& Bloem, I. (1999). Dolphin facilitates and hampers the translation of shark: An effect of context modality. Poster presented at the Nederlandse Vereniging voor Psychonomie, Egmont aan Zee, The Netherlands.

Levelt, W. J. M. (1989). Speaking. From intention to articulation. Cambridge: MIT Press.

Levelt, W. J. M., Praamstra, P., Meyer, A. S., Helenius, P., \& Salmelin, R. (1998). A MEG study of picture naming. Journal of Cognitive Neuroscience, 10, 553-576.

Levelt, W. J. M., Roelofs, A., \& Meyer, A. S. (1999). A theory of lexical access in speech production. Behavioral and Brain Sciences, 22, 1-75.

Levelt, W. J. M., Schriefers, H., Vorberg, D., Meyer, A. S., Pechmann, T., \& Havinga, J. (1991). The time course of lexical access in speech production: A study of picture naming. Psychological Review, 98, 122-142.

Maeß, B., Friederici, A. D., Damian, M., Meyer, A. S., \& Levelt, W. J. M. (2002). Semantic category interference in overt picture naming: Sharpening current density localization by PCA. Journal of Cognitive Neuroscience, 14, 455-462.

Peterson, R. R., \& Savoy, P. (1998). Lexican selection and phonological encoding during language production: Evidence for cascaded processing. Journal of Experimental Psychology. Learning, Memory, and Cognition, 24, 539-557.

Praamstra, P., Meyer, A. S., \& Levelt, W. J. M. (1994). Neurophysiological manifestations of phonological processing: Latency variation of a negative ERP component timelocked to phonological mismatch. Journal of Cognitive Neuroscience, 6, 204-219.

Praamstra, P., \& Stegeman, D. F. (1993). Phonological effects on the auditory N400. Cognitive Brain Research, 1, 73-86.

Radeau, M., Besson, M., Fonteneau, E., \& Castro, S. L. (1998). Semantic, repetition and rime priming between spoken words: Behavioral and electrophysiological evidence. Biological Psychology, 48, 183-204.

Roelofs, A. (1992). Lemma retrieval in speaking. A theory, computer simulations, and empirical data. Doctoral dissertation, Universität Nijmegen, Nijmegen, Niederlande.

Rugg, M. D. (1984a). Event-related potentials in phonological matching tasks. Brain and Language, 23, 225-240.

Rugg, M. D. (1984b). Event-related potentials and the phonological processing of words and non-words. Neuropsychologica, 22, 435-443.

Rugg, M. D., \& Barrett, S. E. (1987). Event-related potentials and the interaction between orthographic and phonological information in a rhyme-judgment task. Brain and Language, 32, 336-361.

Schmitt, B. M., Münte, T. F., \& Kutas, M. (2000). Electrophysiological estimates of the time course of semantic and phonological encoding during implicit picture naming. Psychophysiology, 37, 473-484.

Van Petten, C., Coulson, S., Rubin, S., Plante, E., \& Parks, M. (1999). Time course of word identification and semantic integration in spoken language. Journal of Experimental Psychology. Learning, Memory, and Cognition, 25, 394-417.

van Turennout, M., Hagoort, P., \& Brown, C. M. (1997). Electrophysiological evidence on the time course of semantic and phonological processes in speech production. Journal of Experimental Psychology. Learning, Memory, and Cognition, 23, 787-806.

van Turennout, M., Hagoort, P., \& Brown, C. M. (1998). Brain activity during speaking: From syntax to phonology in 40 milliseconds. Science, 280, 572-574.

Vigliocco, G., Vinson, D. P., Martin, R. C., \& Garrett, M. F. (1999). Is "count" and "mass" information available when noun is not? An investigation of tip of the tongue states and anomia. Journal of Memory and Language, 40, 534-558.

Wohlert, A. B. (1993). Event-related brain potentials preceding speech and nonspeech oral movements of varying complexity. Journal of Speech and Hearing Research, 36, $897-905$. 